I. M. Okhapkin

A. R. Khokhlov

E. E. Makhaeva

\section{Two-dimensional classification of amphiphilic monomers based on interfacial and partitioning properties. 1. Monomers of synthetic water-soluble polymers}

Published online: 22 August 2006

C) Springer-Verlag 2006
The online version of the original article can be found at http://dx. doi.org/10.1007/s00396-005-1342-1 of the article with published errors.

I. M. Okhapkin · A. R. Khokhlov ( $\bowtie)$

Department of Polymer Science, University of Ulm,

Albert-Einstein-Allee 11,

89069 Ulm, Germany

e-mail: khokhlov@polly.phys.msu.ru

Tel.: +7-095-9392959

Fax: +7-095-9392988

I. M. Okhapkin · A. R. Khokhlov

Nesmeyanov Institute of Organoelement Compounds,

Vavilov Street 28,

119991 Moscow, Russia

A. R. Khokhlov · E. E. Makhaeva

Physics Department,

Moscow State University,

119992 Moscow, Russia

\section{Colloid Polym Sci (2005) 284:117-123}

On page 120, Fig. 4 is missing as, unfortunately, Fig. 5 was published twice. The correct Fig. 4 is the following. The publisher apologizes for this mistake.

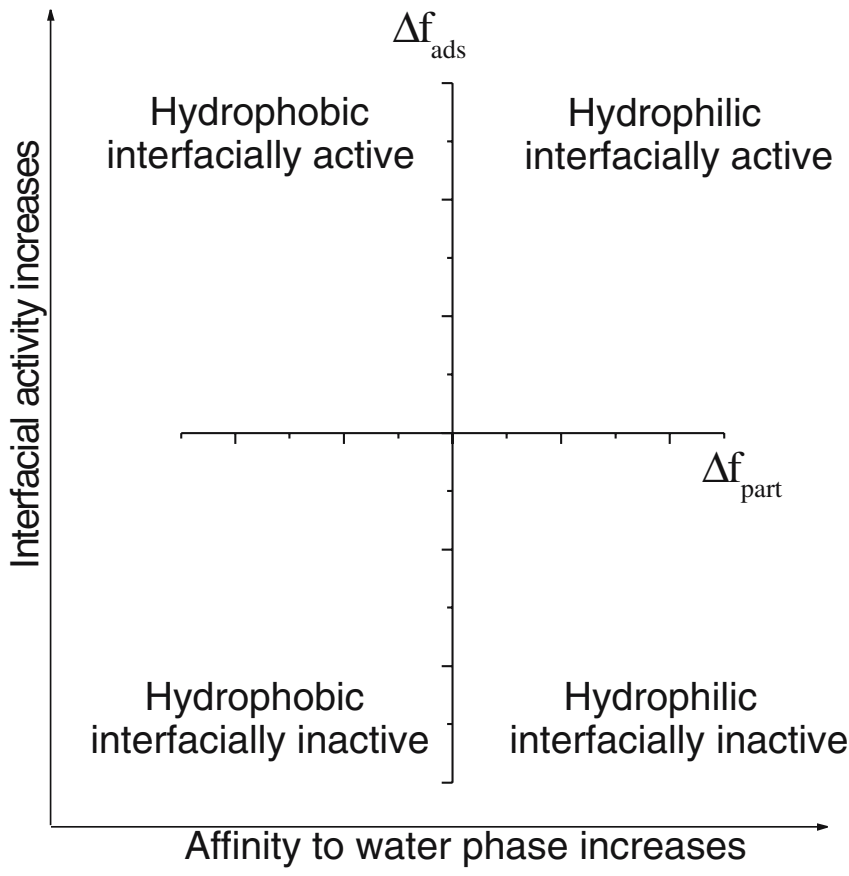

Fig. 4 Two-dimensional diagram of interfacial activity and phase affinity, general view 